\section{Assessment of Motor Cortex in Active, Passive and Imagery Wrist Movement Using Functional MRI}

\author{
Hamid Sharini ${ }^{1 \oplus}$, Shokufeh Zolghadriha², Nader Riyahi \\ Alam ${ }^{3,4,5 * \odot, ~ M a z i a r ~ J a l a l v a n d i ², ~ H a m i d ~ K h a b i r i ³ ~}{ }^{3}$, Hossein \\ Arabalibeik ${ }^{3,6}$, Mohadeseh Nadimi²
}

\begin{abstract}
Background: Functional Magnetic resonance imaging (fMRI) measures the small fluctuation of blood flow happening during task-fMRI in brain regions.

Objective: This research investigated these active, imagery and passive movements in volunteers design to permit a comparison of their capabilities in activating the brain areas.

Material and Methods: In this applied research, the activity of the motor cortex during the right-wrist movement was evaluated in 10 normal volunteers under active, passive, and imagery conditions. $\mathrm{T} 2 *$ weighted, three-dimensional functional images were acquired using a BOLD sensitive gradient-echo EPI (echo planar imaging) sequence with echo time (TE) of $30 \mathrm{~ms}$ and repetition time (TR) of $2000 \mathrm{~ms}$.

The functional data, which included 248 volumes per subject and condition, were acquired using the blocked design paradigm. The images were analyzed by the SPM12 toolbox, MATLAB software.

Results: The findings determined a significant increase in signal intensity of the motor cortex while performing the test compared to the rest time $(\mathrm{p}<0.05)$. It was also observed that the active areas in hand representation of the motor cortex are different in terms of locations and the number of voxels in different wrist directions. Moreover, the findings showed that the position of active centers in the brain is different in active, passive, and imagery conditions.
\end{abstract}

Conclusion: Results confirm that primary motor cortex neurons play an essential role in the processing of complex information and are designed to control the direction of movement. It seems that the findings of this study can be applied for rehabilitation studies.

Citation: Sharini H, Zolghadriha Sh, Riyahi Alam N, Jalalvandi M, Khabiri H, Arabalibeik H, Nadimi M. Assessment of Motor Cortex in Active, Passive and Imagery Wrist Movement Using Functional MRI. J Biomed Phys Eng. 2021:11(4):515-526 doi: 10.31661/jbpe.v0i0.1034.

\section{Keywords}

Functional MRI; Active Movement; Passive Movement; Imaginary Movement; Motor Cortex; Rehabilitation; Brain-Computer Interfaces; Wrist Movement

\section{Introduction}

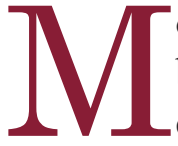

otor disability is one of the most prevalent impairments caused by brain injury. Disables are remedied by rehabilitation exercises. However, these methods require some left abilities in the injured organ of the body making these physical exercises impossible for disables suffering from the nearly complete loss of motor func-
${ }^{1} \mathrm{PhD}$, Department of Medical Physics and Biomedical Engineering, School of

Medicine, Kermanshah University of Medical Sciences (KUMS), Kermanshah, Iran

${ }^{2} \mathrm{MSc}$, Department of Medical Physics and Biomedical Engineering, School of

Medicine, Tehran Univer-

sity of Medical Sciences

(TUMS), Tehran, Iran

${ }^{3} \mathrm{PhD}$, Department of Medical Physics and Biomedical

Engineering, School of

Medicine, Tehran Univer-

sity of Medical Sciences

(TUMS), Tehran, Iran

${ }^{4} \mathrm{PhD}$, PERFORM Center,

Preventive Medicine and

Personal Health Care

Center, Concordia Univer-

sity, Montreal, Quebec,

Canada

${ }^{5} \mathrm{PhD}$, Medical Pharma-

ceutical Sciences Re-

search Center (MPRC), the

institute of Pharmaceutical

Sciences, Tehran Univer-

sity of Medical Sciences,

Tehran, Iran

${ }^{6} \mathrm{PhD}$, Research Center for

Science and Technology in

Medicine (RCSTM), Tehran

University of Medical Sci-

ences (TUMS), Tehran, Iran

*Corresponding author:

Nader Riyahi Alam

Department of Medical

Physics and Biomedical

Engineering, Faculty of

Medicine, Tehran Univer-

sity of Medical Sciences

(TUMS), Tehran, Iran

E-mail: riahinad@sina.

tums.ac.ir

Received: 1 October 2018

Accepted: 17 October 2018 
tion. Thus, alternative methods are required to help the recovery of the motor system without the need for a distinct behavior. Given the assumption that improvement is supported by activation of the motor function [1-2], the question appears how such activation may be produced without real apparent motions. For this purpose, at least three methods have been proposed as follows:

One method is motor imagery training, which is the mental visualization of a motor practice, to activate the sensorimotor cortex in the brain without performing motion. It has been proposed that kinetic motor imagery includes a similar neural network such as motor planning [3-4], which relies on similar motor structures, such as motor implementation [56]. Moreover, motor imagery has several similarities with movement execution, including behavioral [7] and physiological parameters [8] and importantly, the functional neuroanatomical correlations [9-12]. In addition, mental exercise, which is learning with motor imagery, has been widely used in sports training and rehabilitation [9-20]. From three proposed procedures, motor imagery training is possibly the most extensively applied procedure in rehabilitation due to its successful applications in scientific training of sports [9].

The second method is passive movement that the physiotherapist passively moves the injured hand. Passive movement is believed to actuate the sensorimotor cortex through different transporting proprioceptive information not only to sensory but also to motor cortex function [20-25]. Earlier researches demonstrated that brain networks' connectivity of passive movement and active motion overlap strongly [26]. This evidence proposes that passive motion can be used effectively in rehabilitation [26-30].

Movement execution is the third procedure that motion execution activates the human network brain, which includes neurons reacting to the obvious execution of a movement [3033]. The evidence proposes that movement execution constantly is used in rehabilitation procedures [34].

All three movement modes including, passive, imagery, and active movement execution, are to stimulate the motor cortex in order to make the methods appropriate for the clinical neurologic intervention, the question arises that how these methods directly affect each other. Such information is significant for developing and correcting covert motion interfaces optimized for disabling factors such as motor damage.

Many papers support the general opinion of covert motion methods. However, the majority of these research projects considered only one method of covert movement or, at most, compared two methods of motor imagery and passive method [34-39]. For this reason, conclusions between researches have to be performed for comparison for all methods of covert movement.

Current research, therefore, aimed to solve this shortcoming by simultaneously analyzing all three motion techniques in a repeated experiment design to discover the similarities and differences in their neuroanatomical effects.10 healthy subjects participated in this study.

\section{Material and Methods}

In this applied research, the fMRI data was analyzed using GLM to detect the activation of brain regions in different motion methods in order to create an interface for rehabilitation in healthy subjects. Before describing these methods, we should describe the appropriate model structure more particularly. In this study, we focused on several regions of the motor cortex because these areas were related to motor rehabilitation.

\section{Participants}

The study comprised ten healthy, righthanded subjects, including 6 males and 4 females with a mean age of 27 years. Written consent was obtained from all volunteers be- 
Brain Activity during Wrist Movement for $\mathrm{BCl}$

fore participating in the study. The protocol of the human research was adopted by the local ethical committee, Tehran University of Medical Sciences (TUMS), Tehran, Iran (Approval number: IR.TUMS IR.TUMS.MEDICINE. REC.1395.1018).

\section{Task}

Volunteers laid down in a magnetic resonance imaging (MRI) system in the resting position. All participants performed a visual step-tracking task by moving their right wrist in four directions, including up, down, right, left. To maximize subjects' comfort and fix their arm and forearm, we used Antimicrobial Positioning Pads, which also facilitated doing the task by subjects (Figure 1). Before each test, we ensured that participants could move their wrist freely in all directions. The directions, which participants should move their wrist towards them, were presented using MRI-compatible glasses.

Before starting the test, subjects saw a blank page, which "ready" was written on it, and at that moment, they should keep their wrist in a natural position without rotation and movement. There were three conditions, including active, imagery, and passive. For the active condition as soon as the blank page disappeared, participants should move their wrist toward directions shown them (Figure 2A). After each step of moving toward a direction, a move to the center was displayed. This caused the wrist to return to the natural position. For imagery condition, volunteers were trained to visualize moving toward the direction shown in Figure 2B for passive condition, volunteers were trained to relax their wrist and allow it to move freely by examiner with close eyes.

Regarding the wrist position, movements to up, down, right and left corresponded to radial, ulnar, extension, and flexion movements, respectively. Participants were trained to move their wrist to the directions as fast as possible and keep their hands in that directions until the center box appear. The remaining time in each direction was 3 seconds, and in the center was a random number of 2,3 , or 4 seconds. Therefore, each step-racking movement lasted between 5, 6, or 7 seconds randomly, and there

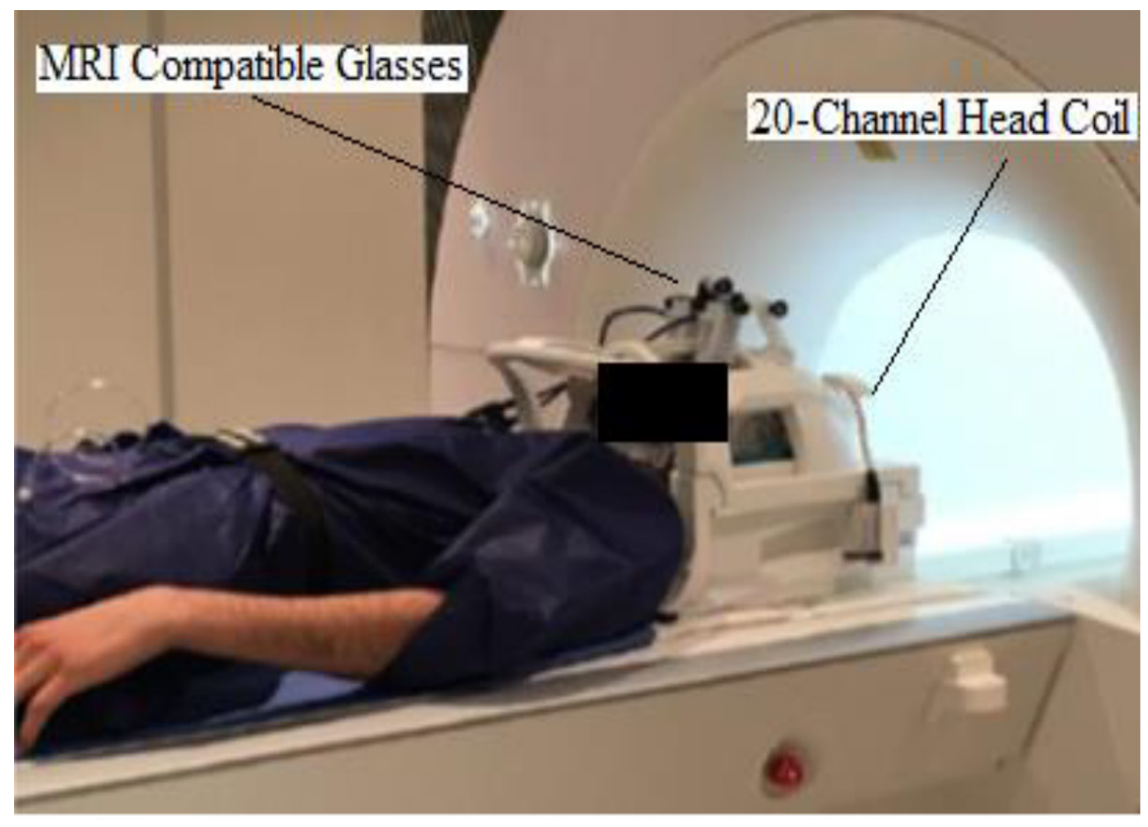

Figure 1: Subjects head positioning inside a standard twenty channels head coil. Arm and forearm were fixed with antimicrobial positioning pads and directions were presented using magnetic resonance imaging (MRI) compatible glasses. 
A)
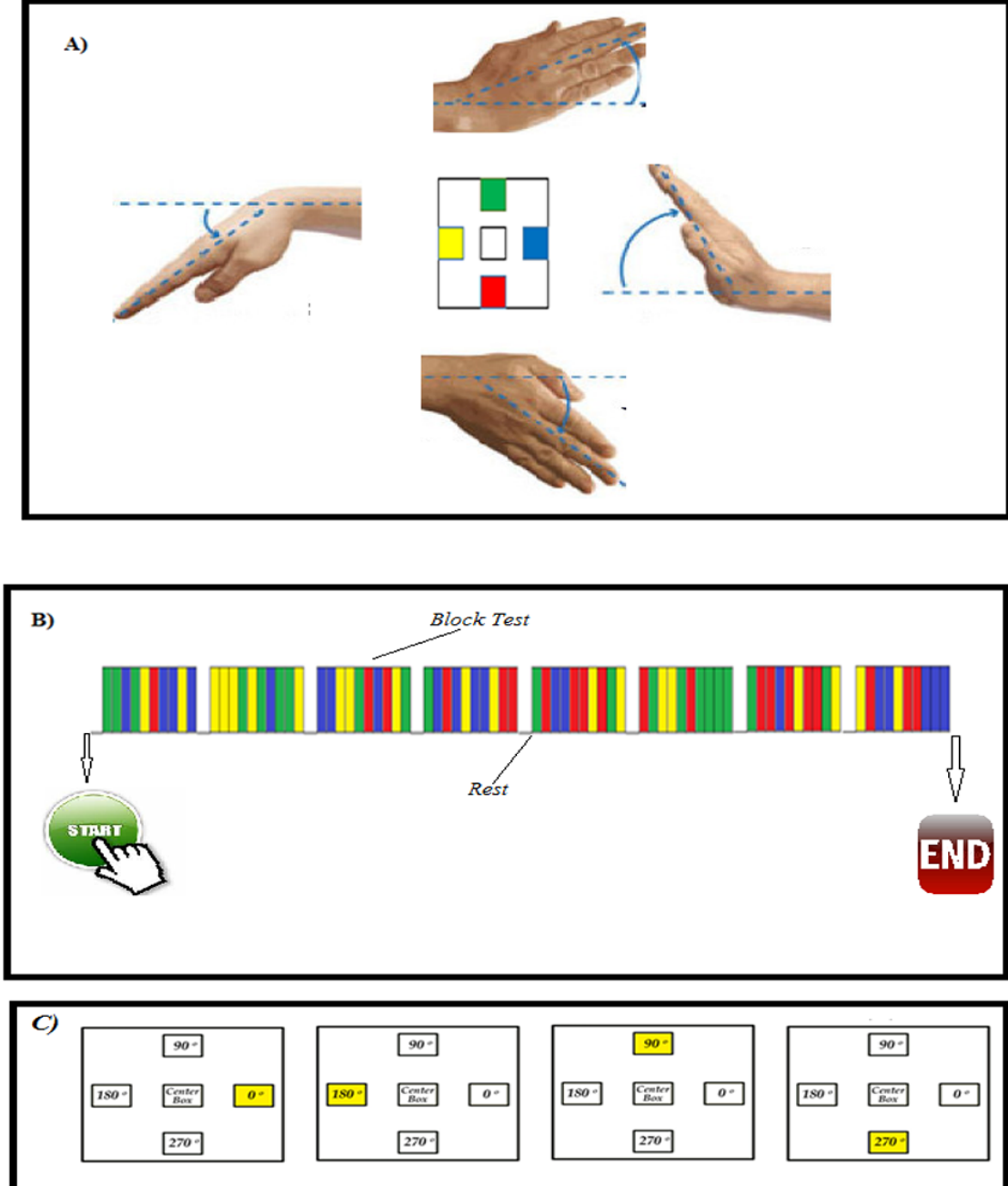

Figure 2: A) The four directional stimuli.B) Block design in Functional Magnetic resonance imaging (fMRI) experiment. The 8-block test that each block included ten moves and randomly 20 times were pointed out to each direction. C) Schematic overview of the four directional stimuli. Step tracking was performed by moving the wrist into the four directions, including right (ulnar deviation), left (radial deviation), up (extension) and down (flexion).

was a rest of 4 seconds after all ten movements. During this test, every direction was mentioned 20 times (Figure 2C). Examiner controlled the correctness of wrist movements of subjects during the test, and whenever was needed, participants were given auditory and visual feedback between task blocks.

\section{MRI characteristics}

Imaging was carried out at the national brain-mapping lab, Tehran, Iran. Volunteers laid down in the MRI system, and the head coil was used to decrease head motion and increase the signal to noise (SNR). Data were acquired applying a 3 Tesla magnetic field (Siemens, MAGNETOM Prisma) with a standard 20 channel head coil. T2* weighted, three-dimensional functional images were acquired applying a BOLD sensitive gradient echo and echo-planar imaging (EPI) sequence with echo time (TE) of $30 \mathrm{~ms}$ and repetition time (TR) of $2000 \mathrm{~ms}$. Through each TR, 34 axial slices were obtained $\left(90^{\circ}\right.$ flip angle, $64 * 64$ matrix size, $210 \mathrm{~mm}$ FOV, $3 \times 3 \times 3 \mathrm{~mm}$ 
Brain Activity during Wrist Movement for $\mathrm{BCl}$

voxel size). The functional scans contained 248 volumes per subject. With high resolution, whole-brain images were obtained from each volunteer applying a T1 weighted MPRAGE sequence (TR $2300 \mathrm{~ms}$, TE $2.97 \mathrm{~ms}, 11^{\circ}$ flip angle, 176 slices, $256 \times 256 \mathrm{~mm}$ FOV, $1 \times 1 \times 1$ $\mathrm{mm}$ voxel size).

\section{General Linear Model (GLM) and fMRI Data}

GLM is a powerful approach for fMRI data analysis and mathematically equivalent to multiple regression [39]. This method has the strength of integrating multiple qualitative and quantitative independent variables and can be expressed using the matrix form, including predictors, voxel time course, the beta values, and the residuals (Eq.1):

$$
\left[\begin{array}{c}
y_{1} \\
\vdots \\
y_{2}
\end{array}\right]=\left[\left[\begin{array}{ccc}
1 & X_{11} \cdots & X_{1 p} \\
\vdots & \ddots & \vdots \\
1 & X_{n 1} \cdots & X_{n p}
\end{array}\right]\left[\begin{array}{c}
b_{0} \\
\vdots \\
b_{p}
\end{array}\right]+\left[\begin{array}{c}
e_{1} \\
\vdots \\
e_{n}
\end{array}\right]\right.
$$

$\mathrm{y}$ : Voxel time course

$\mathrm{X}$ : Predictors

b: Beta values

e: residuals

All conditions, including right, left, up, down and rest were modeled as predictors in the fMRI design matrix. The four beta weights of B1, B2, B3, and B4 would be interpreted as decreasing and increasing activity relative to the modeled baseline signal level (BSL) using the constant term (CT) (Figure 3 ) regardless of the rest condition.

\section{Data analysis}

Statistical analyses and processing of data were done by SPM12 toolbox (http://www.fil. ion.ucl.ac.uk/spm/software/spm12). The preprocessing step included field map correction, realignment, and co-registration of functional and anatomical scans, segmentation, normalization, and smoothing. First, we used field map toolbox to correct the signal changes due to the non-uniformity of the magnetic field. Second, for motion correction step, all functional volumes were spatially realigned to the mean functional volume. At the end of this stage, the position of the brain during the time was identical for all volumes. Next, unwrapped mean EPI image was co-registered to the participant's T1-weighted anatomical image. In the fourth stage, segmentation was used for bias correction. Then, both anatomical and functional images were normalized

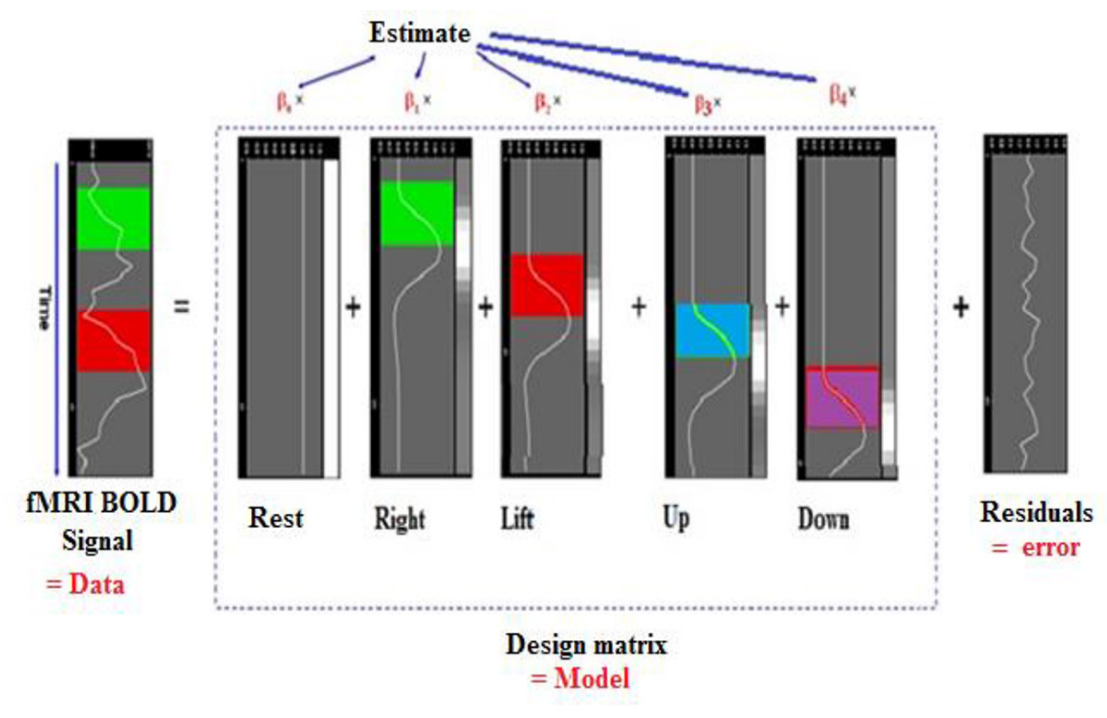

Figure 3: General Linear Model (GLM) of task experiment to model the movement of the wrist in the right, left, up and down directions. 
to the template of the Montreal Neurological Institute (MNI), and at the final stage, normalized functional images were smoothed by applying a Gaussian filter with $8 \mathrm{~mm}$ full width at half maximum (FWHM).

Brain activations were calculated according to the standard statistical procedures in SPM12. The analysis was conducted at two levels, including first level analysis and group level analysis. In the first level analysis, a map of brain activity for each person was obtained through applying linear multiple regression models with 4 event-related regressors. Then, in the group-level analysis, we applied ANOVA with four levels (matching with the four directions) to define the differences and similarities among the group and study cerebral activation patterns associated with each direction. We evaluated maximally activated voxels per direction. We used $p=0.001$ for limiting the number of false positive voxels.

\section{Extraction of number voxels from each Boardman area}

In the early $1900 \mathrm{~s}$, into 52 areas, which were determined and enumerated by the German anatomist Korbinian Brodmann, the Brod- mann areas of the cerebral cortex were determined by the histological structure and cellular organization. We selected six Brodmann areas, including areas $3,1 \& 2$ - primary somatosensory cortex, area 4 - primary motor cortex, area 5 - somatosensory association cortex, area 6 - premotor cortex and supplementary motor cortex to evaluate brain activity (Figure 4) [40-43].

The SPM toolbox extracts the voxels of Boardman regions rather than the average values, as the earlier approach is more robust to the inconsistency of response within a cluster. The average value can be considered as a particular case of the voxels of Boardman regions if the corresponding Boardman voxels are in a cluster equally.

\section{Results}

Activation caused by motion in different directions, specified by the sensory cues received by the eye, is simply demonstrated as activation relevant to that direction. Centers for maximum activation were estimated in primary motor cortex (M1) related to the Brodmann area (BA) 4. Right-hand motion, irrespective of its direction, excited brain ac-

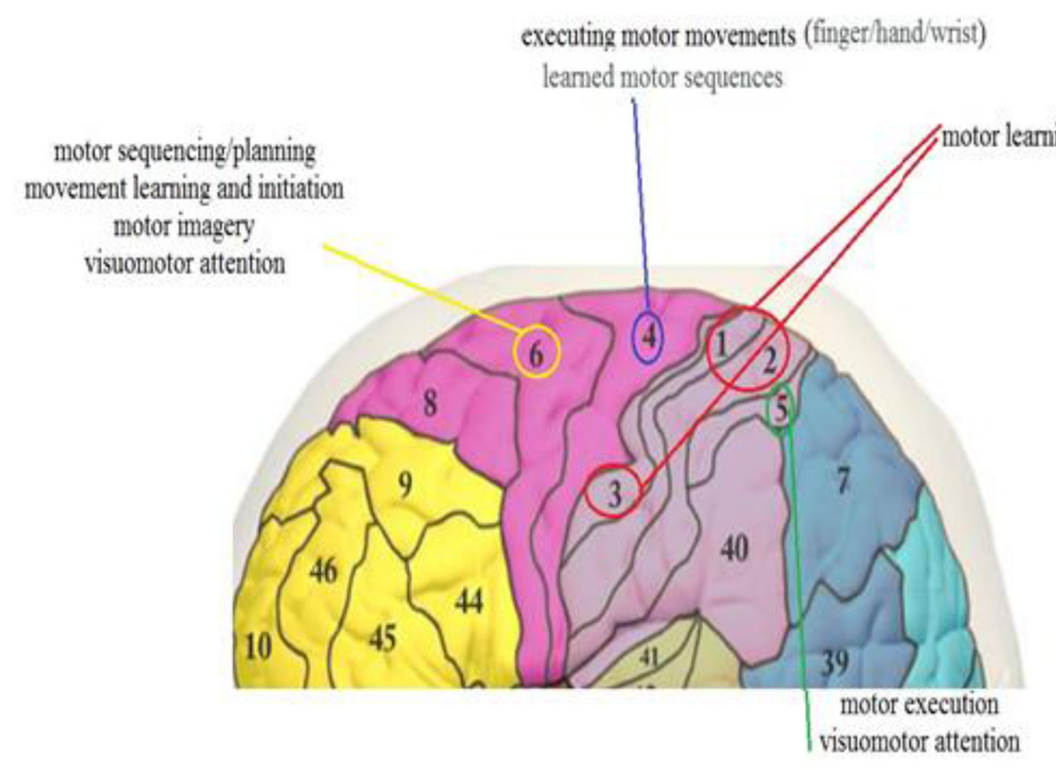

Figure 4: The Brodmann area of movement is the region of the motor cortex in the human brain including area 1-6. 
Brain Activity during Wrist Movement for $\mathrm{BCl}$

tivation in the S1 somatotopic representation of the hand in contralateral M1 [12-14]. Nevertheless, there were apparent variances in the focus of maximum brain activation among the four primary directions, including $0,90,180$, and 270 (Figures 5, 6 and 7).

\section{Cortical Activation Associated} with Active Movements

Activation relevant to motion in direction $0^{\circ}$ was most laterally and extended into the cortical convexity, while directions of $180^{\circ}$ and $270^{\circ}$ demonstrated activation more centrally within the hand region. The $270^{\circ}$ direction demonstrated activation more ventrally than the other directions, while activation related to the $90^{\circ}$ direction was more dorsally (Figure 5).

\section{Cortical Activation Associated} with Passive Movements

Activation relevant to motion in direction $180^{\circ}$ was located more laterally and extended into the cortical convexity, while directions $0^{\circ}$ and $90^{\circ}$ demonstrated activation more centrally within the hand region. The 180 direction demonstrated activation more ventrally than the other directions while activation relevant to the $270^{\circ}$ direction was more dorsally in the M1 area (Figure 6).

\section{Cortical Activation Associated}

\section{with Imagery Movements}

Activation relevant to motion in direction $270^{\circ}$ was more laterally and extended into the cortical convexity, while directions $90^{\circ}$ and $180^{\circ}$ demonstrated activation more centrally within the hand region. The $270^{\circ}$ direction demonstrated activation more ventrally than the other directions while activation relevant to the $0^{\circ}$ direction was more dorsally in the M1 area (Figure 7).

The number of increased activated voxels as a function of Motor Cortex in active, passive and imaginary wrist movement is shown in Table 1.
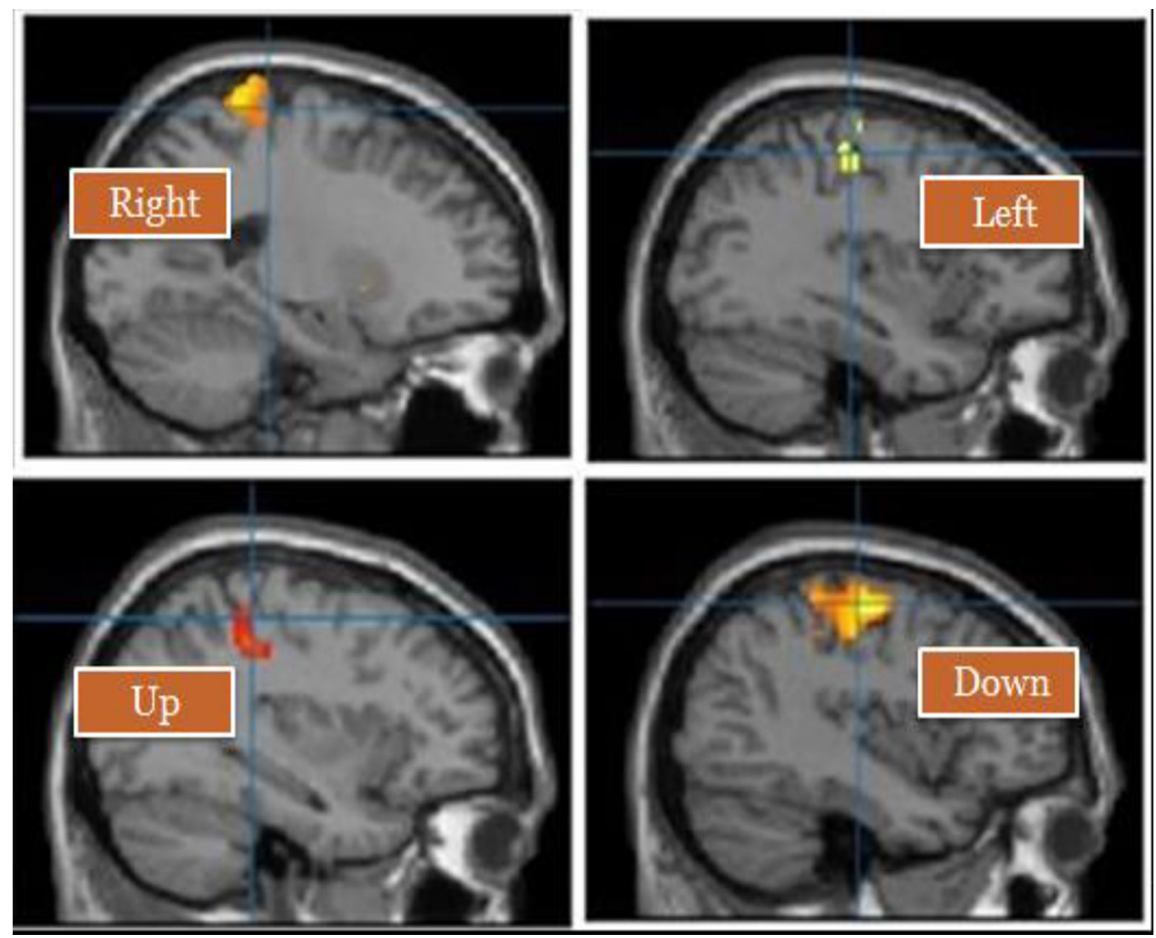

Figure 5: Direction relevant activation patterns in the primary motor region in active condition. Activated region is shown in the direction of the right, left, up and down during active movement. 

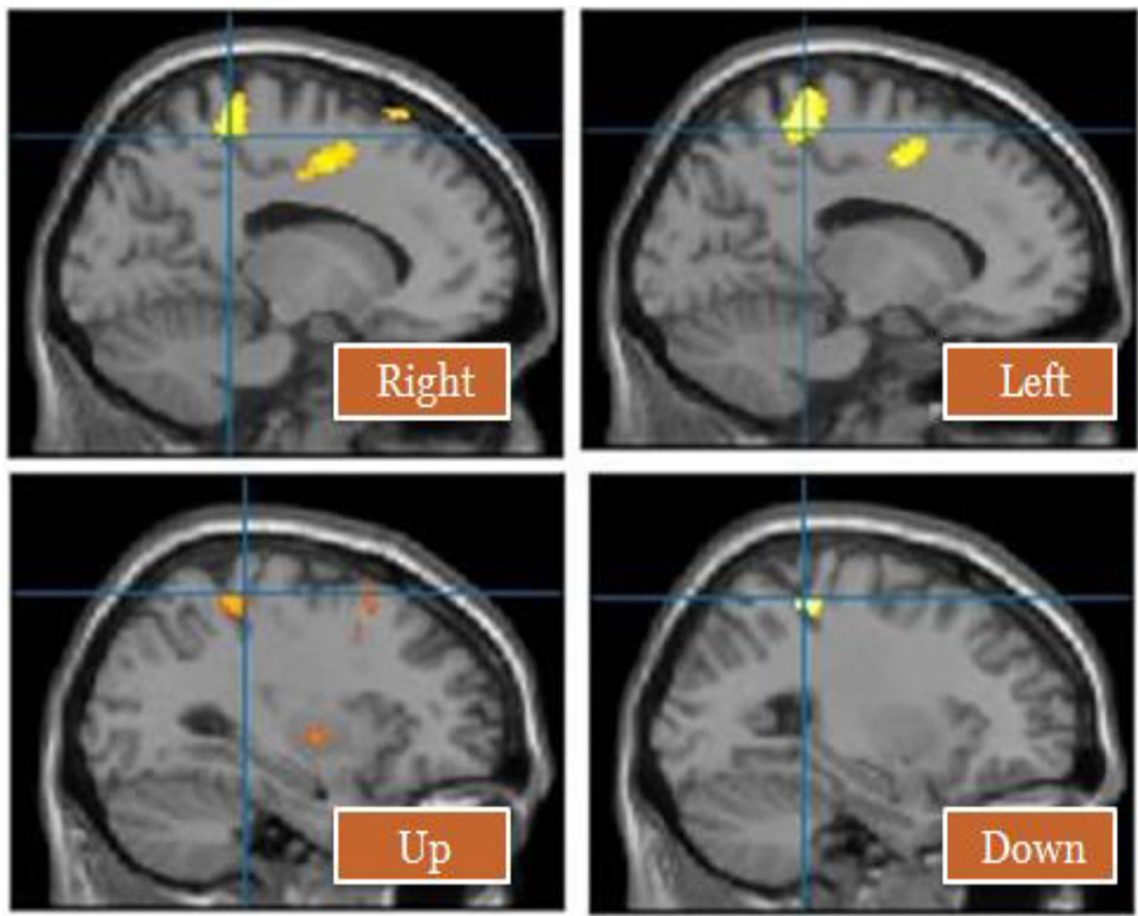

Figure 6: Direction- relevant activation patterns in the primary motor region in passive condition. Activated area is shown in the direction of the right, left, up, down during passive movement.
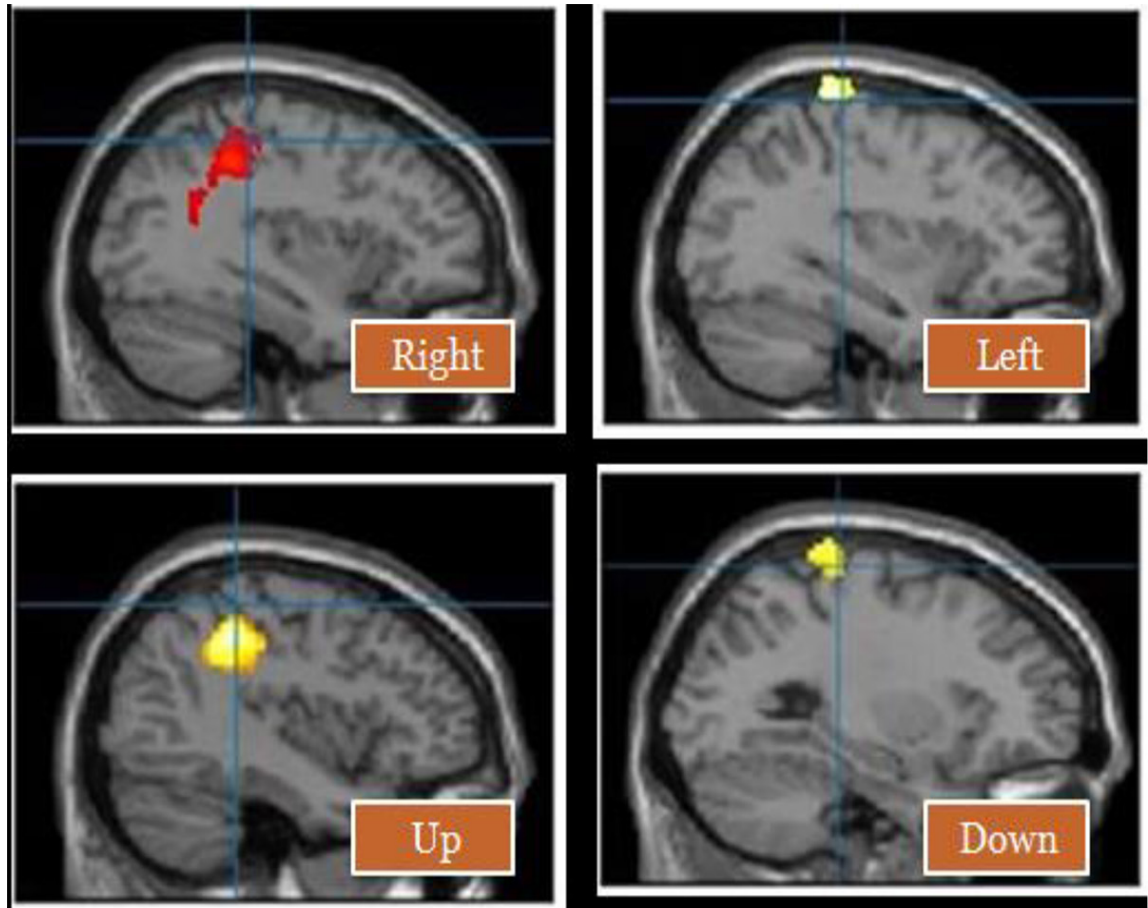

Figure 7: Direction-related activation patterns in the primary motor area in imagery condition. Activated area is shown in the direction of the right, left, up and down during imagery movement. 
Table 1: Statistical parameters relevant to activation patterns in the primary motor region in three conditions.

\begin{tabular}{cccc}
$\begin{array}{c}\text { Broadman } \\
\text { region }\end{array}$ & $\begin{array}{c}\text { Active } \\
\text { movement }\end{array}$ & $\begin{array}{c}\text { Passive } \\
\text { movement }\end{array}$ & $\begin{array}{c}\text { Imagery } \\
\text { movement }\end{array}$ \\
\hline 1 & 8 & 5 & 10 \\
\hline 2 & 60 & 45 & 55 \\
\hline 3 & 140 & 120 & 123 \\
\hline 4 & 133 & 152 & 117 \\
\hline 5 & 26 & 20 & 33 \\
\hline 6 & 104 & 124 & 136
\end{tabular}

\section{Discussion}

This fMRI research demonstrates that activation relevant to the performed motion in particular directions is spatially disjoint in the motor cortex. The results of this research are in agreement with recent researches applied single-cell recordings in monkeys in which populations of neurons in M1 were directionally distinguishable [44-49]. The latest fMRI researches also investigated the neuronal representation of direction in humans [50-51]. This research confirms other researchers' reports about the capability of fMRI and BOLD contrast in obtaining neuronal activity in diverse brain regions. Hence, the present result of direction-relevant activation in the human M1 hand region increases with primary motor cortex (M1) functions; aside from somatotopic ordered actuator system, the primary motor cortex (M1) is also involved in higher-order processing of information.

In order to improve the accuracy of the results and prevent recording false active regions in the cerebral cortex, we used all the scientific principles in different stages of image processing, including head motion correction, which should be avoided during the experiment. Studies done by researchers have determined that the shape of the HRF curve extracted from different regions of the cerebral cortex after physical exercise in normal people has a similar or less similar pattern. Findings proved that while all covert motion methods activated the sensorimotor region, there were apparent differences between healthy and disabled subjects in different modes. In healthy subjects, the pattern of neural activation in obvious performance was best documented by passive motion, followed by motor imagery mode.

The advantage of this study compared to previous studies is experimenting with three active, passive, and imagery conditions. Previously, this study was only performed in active mode, and there was a doubt that whether separate activity centers in the M1 region were created only by activation of different muscles during the test or the movements of the eye during the observation of the target. However, testing in passive and imagery states showed that M1 neurons, along with motion control neurons of different parts of the body, are essential in complex information processing and adjusting control of the movement in the right, left, up and down directions.

The fMRI technique is beneficial for these researches due to its repeatability, sensitivity, and robustness while providing whole-brain analysis and the explanation of signal variations in affected regions relative to those in other brain areas. The fMRI application in the development of such intermediaries may provide essential information on spatiotemporal variations in the neurovascular functioning of an at-risk limb. Our finding of the mechanisms of subacute injury permits the expansion of fMRI imaging for secure and efficient rehabilitation.

\section{Conclusion}

We investigated the activity of the motor cortex during the active, passive, and imagery conditions for the right wrist movement. The clinical feasibility applications and the possible further development of GLM model have been introduced for rehabilitation assessment utilities. This model can be applied for the identification of the neuroplasticity of cortical 
reorganization following focal brain injuries. Therefore, we propose to utilize our method to build a more comprehensive motor mapping in clinical injuries and further steps of stroke recovery treatment studies, as well.

These results provide new information regarding the neural control of hand movements in humans. Our results suggest that the fMRI technique may provide a suitable method for investigating rehabilitation and BCI studies, bypassing some of the normal limitations of brain imaging. The current results suggest that hand movements in humans are controlled bilaterally in the M1. Future studies may use similar methods to investigate hand movements in other directions, as well as created a direct communication pathway between an enhanced or wired brain and an external device.

\section{Acknowledgment}

This research was supported in part by the Research Deputy of Tehran University of Medical Sciences (TUMS), project grant No. of (95-02-30-30034). The authors wish to thank Tehran University of Medical Sciences for the financial and instrumental aid of this study, and National Brain Mapping Laboratory (NBML), Tehran-Iran, for providing the data acquisition.

\section{Conflict of Interest}

None

\section{References}

1. Johnson-Frey SH. Stimulation through simulation? Motor imagery and functional reorganization in hemiplegic stroke patients. Brain Cogn. 2004;55(2):328-31. doi: 10.1016/j. bandc.2004.02.032. PubMed PMID: 15177807.

2. Kaneko F, Murakami T, Onari K, Kurumadani H, Kawaguchi K. Decreased cortical excitability during motor imagery after disuse of an upper limb in humans. Clin Neurophysiol. 2003;114(12):2397-403. doi: 10.1016/s1388-2457(03)00245-1. PubMed PMID: 14652100.

3. Jeannerod, M. The representing brain: Neural correlates of motor intention and imagery. Behavioral and Brain Sciences. 1994;17(2):187-202. doi: 10.1017/S0140525X00034026.

4. Jeannerod M, Frak V. Mental imaging of motor activity in humans. Curr Opin Neurobiol. 1999;9(6):7359. doi: 10.1016/s0959-4388(99)00038-0. PubMed PMID: 10607647.

5. Li M, Liu Y, Wu Y, Liu S, Jia J, Zhang L. Neurophysiological substrates of stroke patients with motor imagery-based Brain-Computer Interface training. Int J Neurosci. 2014;124(6):403-15. doi: 10.3109/00207454.2013.850082. PubMed PMID: 24079396.

6. Munzert J, Zentgraf K, Stark R, Vaitl D. Neural activation in cognitive motor processes: comparing motor imagery and observation of gymnastic movements. Exp Brain Res. 2008;188(3):437-44. doi: 10.1007/s00221-008-1376-y. PubMed PMID: 18425505.

7. Decety J, Jeannerod M. Mentally simulated movements in virtual reality: does Fitts's law hold in motor imagery? Behav Brain Res. 1995;72(1-2):12734. doi: 10.1016/0166-4328(96)00141-6. PubMed PMID: 8788865.

8. Kranczioch C, Mathews S, Dean PJ, Sterr A. On the equivalence of executed and imagined movements: evidence from lateralized motor and nonmotor potentials. Hum Brain Mapp. 2009;30(10):327586. doi: $10.1002 / \mathrm{hbm} .20748$. PubMed PMID: 19253343.

9. Szameitat AJ, Shen S, Sterr A. Effector-dependent activity in the left dorsal premotor cortex in motor imagery. Eur J Neurosci. 2007;26(11):3303-8. doi: 10.1111/j.1460-9568.2007.05920.x. PubMed PMID: 18005067.

10. Szameitat AJ, Shen S, Sterr A. Motor imagery of complex everyday movements. An fMRI study. Neurolmage. 2007;34(2):702-13. doi:10.1016/j. neuroimage.2006.09.033.

11. Porro CA, Francescato MP, Cettolo V, Diamond ME, et al. Primary motor and sensory cortex activation during motor performance and motor imagery: a functional magnetic resonance imaging study. $J$ Neurosci. 1996;16(23):7688-98. PubMed PMID: 8922425. PubMed PMCID: PMC6579073.

12. Lotze M, Halsband U. Motor imagery. J Physiol Paris. 2006;99(4-6):386-95. PubMed PMID: 16716573. doi: 10.1016/j.jphysparis.2006.03.012.

13. Braun SM, Beurskens AJ, Borm PJ, Schack T, Wade DT. The effects of mental practice in stroke rehabilitation: a systematic review. Arch Phys Med Rehabil. 2006;87(6):842-52. doi: 10.1016/j. apmr.2006.02.034. PubMed PMID: 16731221.

14. Cramer SC, Lastra L, Lacourse MG, Cohen MJ. 
Brain Activity during Wrist Movement for $\mathrm{BCl}$

Brain motor system function after chronic, complete spinal cord injury. Brain. 2005;128(12):294150. doi: 10.1093/brain/awh648. PubMed PMID: 16246866 .

15. Cramer SC, Orr EL, Cohen MJ, Lacourse MG. Effects of motor imagery training after chronic, complete spinal cord injury. Experimental Brain Research. 2007;177(2):233-42. doi: 10.1007/ s00221-006-0662-9.

16. Feltz DL, Landers DM. The effects of mental practice on motor skill learning 17.and performance: a meta-analysis. J Sport Psychol. 1983;5:25-57.

17. Szynkiewicz SH, et al. Motor Imagery Practice and Increased Tongue Strength: A Case Series Feasibility Report. Journal of Speech, Language, and Hearing Research. 2019;62(6):1676-84. doi: 10.1044/2019_JSLHR-S-18-0128.

18. Jackson PL, Lafleur MF, Malouin F, Richards C, Doyon J. Potential role of mental practice using motor imagery in neurologic rehabilitation. Archives of Physical Medicine and Rehabilitation. 2001;82(8):1133-41. doi: 10.1053/ apmr.2001.24286.

19. Jackson PL, Lafleur MF, Malouin F, Richards $\mathrm{CL}$, Doyon J. Functional cerebral reorganization following motor sequence learning through mental practice with motor imagery. Neuroimage. 2003;20(2):1171-80. doi: 10.1016/S10538119(03)00369-0.

20. Munzert J, Lorey B, Zentgraf K. Cognitive motor processes: the role of motor imagery in the study of motor representations. Brain Research Reviews. 2009;60(2):306-26. doi: 10.1016/j.brainresrev.2008.12.024.

21. Dechaumont-Palacin S, Marque P, De Boissezon X, et al. Neural correlates of proprioceptive integration in the contralesional hemisphere of very impaired patients shortly after a subcortical stroke: an FMRI study. Neurorehabilitation and Neural Repair. 2008;22(2):154-65. doi: $10.1177 / 1545968307307118$.

22. Lemon RN. Neural control of dexterity: what has been achieved? Experimental Brain Research. 1999;128(1-2):6-12. doi: 10.1007/ s002210050811.

23. Lemon RN, Porter R. Afferent input to movementrelated precentral neurones in conscious monkeys. Proceedings of the Royal Society of London. Proc R Soc Lond B Biol Sci. 1976;194(1116):313-39. PubMed PMID: 11491.

24. Naito E, Roland PE, Ehrsson HH. I feel my hand moving: a new role of the primary motor cortex in somatic perception of limb movement. Neu- ron. 2002;36(5):979-88. doi: 10.1016/S08966273(02)00980-7.

25. Terumitsu M, Ikeda K, Kwee IL, Nakada T. Participation of primary motor cortex area $4 \mathrm{a}$ in complex sensory processing: 3.0-T fMRI study. Neuroreport. 2009;20(7):679-83. doi: 10.1097/ WNR.0b013e32832a1820.

26. Mima T, Sadato N, Yazawa S, Hanakawa T, Fukuyama $\mathrm{H}$, Yonekura $\mathrm{Y}$, Shibasaki H. Brain structures related to active and passive finger movements in man. Brain. 1999;122(10):1989-97. doi: 10.1093/ brain/122.10.1989.

27. Krakauer JW. Motor learning: its relevance to stroke recovery and neurorehabilitation. Current Opinion in Neurology. 2006;19(1):84-90. doi: 10.1097/01.wc0.0000200544.29915.cc

28. Schmidt RA, Lee TD, Winstein C, Wulf G, Zelaznik HN. Motor control and learning: A behavioral emphasis. Human kinetics; 2018.

29. Stoykov ME, Corcos DM, Madhavan S. Movementbased priming: clinical applications and neural mechanisms. Journal of Motor Behavior. 2017;49(1):8897. doi: 10.1080/00222895.2016.1250716.

30. Lewis GN, Byblow WD. The effects of repetitive proprioceptive stimulation on corticomotor representation in intact and hemiplegic individuals. Clinical Neurophysiology. 2004;115(4):765-73. doi: 10.1016/j.clinph.2003.11.014.

31. Rizzolatti $G$, Sinigaglia C. The functional role of the parieto-frontal mirror circuit: interpretations and misinterpretations. Nature Reviews Neuroscience. 2010;11(4):264-74. doi: 10.1038/nrn2805.

32. Roosink M, Zijdewind I. Corticospinal excitability during observation and imagery of simple and complex hand tasks: implications for motor rehabilitation. Behavioural Brain Research. 2010;213(1):3541. doi: 10.1016/j.bbr.2010.04.027.

33. Jalalvandi M, Riahi Alam N, Sharini $H$, Hashemi $H$, Kohzad S. Optical Imaging of the Motor Cortex in the Brain in Order to Determine the Direction of the Hand Movements Using Functional Near-Infrared Spectroscopy (fNIRS). Iranian Journal of Medical Physics. 2018;15(Special Issue-12th. Iranian Congress of Medical Physics):152. doi: 10.22038/ IJMP.2018.12653.

34. Celnik P, Webster B, Glasser DM, Cohen LG. Effects of action observation on physical training after stroke. Stroke. 2008;39(6):1814-20. doi: 10.1161/STROKEAHA.107.508184.

35. Clark S, Tremblay F, Ste-Marie D. Differential modulation of corticospinal excitability during observation, mental imagery and imitation of hand actions. Neuropsychologia. 2004;42(1):105-12. doi: 
10.1016/S0028-3932(03)00144-1.

36. Filimon F, Nelson JD, Hagler DJ, Sereno MI. Human cortical representations for reaching: mirror neurons for execution, observation, and imagery. Neuroimage. 2007;37(4):1315-28. doi: 10.1016/j. neuroimage.2007.06.008.

37. Iseki K, Hanakawa T, Shinozaki J, Nankaku M, Fukuyama $\mathrm{H}$. Neural mechanisms involved in mental imagery and observation of gait. Neuroimage. 2008;41(3):1021-31. doi: 10.1016/j.neuroimage.2008.03.010

38. Wang C, Wai Y, Weng Y, Yu J, Wang J. The cortical modulation from the external cues during gait observation and imagination. Neuroscience Letters. 2008;443(3):232-5. doi: 10.1016/j.neulet.2008.07.084.

39. Jalalvandi M, Sharini H, Naderi Y, Alam NR. Assessment of Brain Cortical Activation in Passive Movement during Wrist Task Using Functional Near Infrared Spectroscopy (fNIRS). Frontiers in Biomedical Technologies. 2019;6(2):99-105. doi: 10.18502/fbt.v6i2.1691.

40. Piefke M, Kramer K, Korte M, Schulte-Rüther M, Korte JM, Wohlschläger AM, Weber J, Shah NJ, Huber W, Fink GR. Neurofunctional modulation of brain regions by distinct forms of motor cognition and movement features. Human Brain Mapp. 2009;30(2):432-51. doi: 10.1002/hbm.20514.

41. Beckmann CF, Jenkinson M, Smith SM. General multilevel linear modeling for group analysis in FMRI. Neuroimage. 2003;20(2):1052-63. doi: 10.1016/S1053-8119(03)00435-X.

42. Lancaster JL, Woldorff MG, Parsons LM, Liotti $\mathrm{M}$, et al. Automated Talairach atlas labels for functional brain mapping. Human Brain Mapp. 2000;10(3):120-31. doi: 10.1002/10970193(200007)10. PubMed PMID: 10912591.

43. Brett M, Christoff K, Cusack R, Lancaster J. Using the Talairach atlas with the MNI template. Neuroimage. 2001;13(6):85. doi: 10.1016/S10538119(01)91428-4.
44. Georgopoulos AP, Kalaska JF, Caminiti R, Massey JT. On the relations between the direction of twodimensional arm movements and cell discharge in primate motor cortex. Journal of Neuroscience. 1982;2(11):1527-37. doi: 10.1523/JNEUROSCI.02-11-01527.1982.

45. Kakei S, Hoffman DS, Strick PL. Muscle and movement representations in the primary motor cortex. Science. 1999;285(5436):2136-9. doi: 10.1126/ science.285.5436.2136.

46. Caminiti R, Johnson PB, Galli C, Ferraina S, Burnod Y. Making arm movements within different parts of space: the premotor and motor cortical representation of a coordinate system for reaching to visual targets. Journal of Neuroscience. 1991;11(5):118297. doi: 10.1523/JNEUROSCI.11-05-01182.1991.

47. Kakei S, Hoffman DS, Strick PL. Direction of action is represented in the ventral premotor cortex. Nature Neuroscience. 2001;4(10):1020-5. doi: 10.1038/nn726.

48. Oghabian MA, Khosravi HR, Ghiasinejad M, Riahi Alam N. Functional Magnetic Resonance Imaging of Motor Cortex Stimulation Induced by Right Thumb Movement Using 1.5 Tesla Routine Mri System. Jundishapur Scientific Medical Journal. 2003:9-17.

49. Fortier PA, Kalaska JF, Smith AM. Cerebellar neuronal activity related to whole-arm reaching movements in the monkey. Journal of Neurophysiology. 1989;62(1):198-211. doi: 10.1152/ jn.1989.62.1.198.

50. Cowper-Smith CD, Lau EY, Helmick CA, Eskes GA, Westwood DA. Neural coding of movement direction in the healthy human brain. PloS One. 2010;5(10):e13330. doi: 10.1371/journal. pone.0013330.

51. Eisenberg M, Shmuelof L, Vaadia E, Zohary E. Functional organization of human motor cortex: directional selectivity for movement. Journal of Neuroscience. 2010;30(26):8897-905. doi: 10.1523/ JNEUROSCI.0007-10.2010. 